November, 2005

\title{
Hybridization functions of intermetallic Ce compounds with group VI elements
}

\author{
P. Roura-Bas ${ }^{a, *}$, V. Vildosola ${ }^{a, b}$ and A. M. Llois ${ }^{a, b}$ \\ ${ }^{a}$ Departamento de Física, Centro Atómico Constituyentes, Comisión Nacional de Energía \\ Atómica \\ ${ }^{b}$ Departamento de Física, Universidad de Buenos Aires, Buenos Aires, Argentina
}

\begin{abstract}
Among the Cerium compounds, the heavier monochalcogenides $\mathrm{CeY}$ (Y=S,Se,Te) of cerium provide a class of compounds which together with the heavier monopnictides CeX ( $\mathrm{X}=\mathrm{N}, \mathrm{P}, \mathrm{As}, \mathrm{Sb}, \mathrm{Bi}$ ) exhibit extremely interesting physical properties. The hybridization of the partially delocalized f-electrons seems to be responsible for the unusual properties, in particular the magnetic ones which show great chemical sensitivity. As compared to the monopnictides, the monochalcogenides have an additional anion p-electron which shifts the Fermi energy into the p-region of the Cerium 5d-derived density of states, and which should affect the hybridization function of the electrons in the conduction band. In this contribution we analyze the 4 f-hybridization function and the crystal-field splittings of the compounds under study.
\end{abstract}

KEY WORDS: Highly correlated systems, crystal fields, p-electron.

* Corresponding author

CAC-CNEA, Av. Gral. Paz 1499, San Martín (1650), Buenos Aires, Argentina roura@tandar.cnea.gov.ar 
CeY and CeX compounds with $\mathrm{Y}=\mathrm{S}$, Se, Te and $\mathrm{X}=\mathrm{P}, \mathrm{As}, \mathrm{Sb}, \mathrm{Bi}$ are suitable systems to extend the knowledge on the relationship between electronic structure and the physical properties of highly correlated Ce compounds, because they have a simple $\mathrm{NaCl}$ crystal structure. In this systems $\mathrm{Ce}$ ions are in a trivalent state with one $4 \mathrm{f}$ electron. Due to the cubic crystal fields, the $J=5 / 2$ sixfold degenerate magnetic ground state splits into the $\Gamma_{8}$ excited quartet state and the $\Gamma_{7}$ ground state doublet[1]. CeS, CeSe and CeTe exhibit antiferromagnetic transitions at $T_{N}=8.4 \mathrm{~K}, 5.4 \mathrm{~K}$, and $2.2 \mathrm{~K}$, respectively while the CeX compounds have antiferromagnetic transitions at $T_{N}=7.1 \mathrm{~K}, 7.2 \mathrm{~K}$ and $18 \mathrm{~K}$ respectively [2]. There is a small reduction of the crystalfield splittings on going from CeX series to CeY series. The splitting energies between the two $J_{5 / 2}$ states are considerably reduced for the heavier compounds CeTe, CeSb, CeBi [3]. These compounds were studied by N. Kioussis et al [4] by doing first-principle calculations based on the self-consistent, warped-muffin-tin LMTO method, in which only the spherical part of the potential is considered and the $4 f$ state is treated as a corelike one. These authors can explain the experimetal crystal-field splittings calculating the hybridization induced splittings on top of the extrapolated point-charge levels [5].

In this contribution, we study by an alternative method, the decrease of the crystal-field splittings on going from CeX to CeY series and the influence of having an extra $p$-electron in $\mathrm{CeY}$ series as compared to CeX one. We calculate these splittings using an $a b$ initio-many body combined technique. Within the $a b$ initio frame the $4 \mathrm{f}$ state belongs to the valence band and the hybridization function is obtained from the $4 \mathrm{f}-$ projected density of states (DOS) following ref. [6]. We use the all-electron full-potential 
linearized augmented plane wave (FP-LAPW) method as implemented in the Wien-2k code [7] and the local density approximation (LDA) for the exchange and correlation potential. The calculations are perfomed for the systems at their experimental values. The muffin-tin radii, $R_{m t}$ are taken equal to 2.4 a.u. for Ce in all the compounds, and the corresponding radii for the anion ligand varies from 1.6 a.u. to 2.8 a.u. depending on the atomic radius. $102 \mathbf{k}$ points are considered in the irreducible Brillouin zone.

Due to the extra p-electron of the Y-anion in the monochalcogenides, the hybridization function shows that below the Fermi energy $\left(E_{F}\right)$, the $\Gamma_{8}$ symmetry is more strongly hybridized in $\mathrm{CeY}$ than in $\mathrm{CeX}$, as it can be drawn from the comparison of Fig. 1 and Fig. 2. Two thirds of the additional electron in CeY go into the insterstitial region and contributes to the enlarged $\Gamma_{8}$. The ab initio obtained hybridization functions are taken as inputs to calculate the crystal-field splittings within NCA (non crossing approximation) for the infinite- $U$ Anderson model [8]. While in the LDA calculation the $4 f$ state is a valence state, in the NCA-equations, the bare value of the $4 f$ state is taken from experimental photoemission experiments. These values are -3 $\mathrm{eV}$ for $\mathrm{CeX},-2.6 \mathrm{eV}$ for $\mathrm{CeTe}$ and $-2.4 \mathrm{eV}$ for $\mathrm{CeS}$ and CeSe with respect to the Fermi level [3]. Within NCA the self energies and the Green's functions are self-consistently obtained by considering the spectrum up to $6500 \mathrm{~K}$ above the Fermi level for the more delocalized systems ( $\mathrm{Y}=\mathrm{S}, \mathrm{Se}, \mathrm{X}=\mathrm{P}, \mathrm{As}$ ) and up to $5000 \mathrm{~K}$ for the more localized ones ( $\mathrm{Y}=\mathrm{Te}, \mathrm{X}=\mathrm{Sb}$ ). The criterion for choosing the considered energy range was discused in another contribution to this conference [9]. The energy spectrum around the Fermi energy plays an important role in the determination of physical properties such as the 
crystal-field splittings. We obtain the $\Gamma_{7}$ state as the ground state for both series of Ce compounds. The values of the calculated crystal field splittings, $\Delta_{C F}=E\left(\Gamma_{8}\right)-E\left(\Gamma_{7}\right)$ , are in very good agreement with the experimental ones, as shown in table 1 . The crystal-field splittings are of the same order of magnitude in $\mathrm{CeY}$ and in CeX, in fact $\Delta_{C F}(\mathrm{CeY})$ 's are slightly smaller than $\Delta_{C F}(\mathrm{CeX})$ 's both in our results and the experimental data. However, looking at the hybridization functions of the $\Gamma_{7}$ and $\Gamma_{8}$ states and comparing them with the partial $p$ and $5 d$ densities of states shown in the insets of Fig. 1 and Fig. 2, one would expect at first sight a larger splitting for CeY than for CeX due to the much larger $\Gamma_{8}$ hybridization values below $E_{F}$ for the first series than for the second one. From the comparison of the partial densities of states it is clear that below $E_{F}, \Gamma_{8}$ hybridizes essentialy with the $p$-states of the $\mathrm{X}$ and $\mathrm{Y}$ anions, while $\Gamma_{7}$ with the $5 d$-states. Our results indicate that for these Ce compounds, the $\Delta_{C F}$ 's values are determined mainly by the hybridization character near the Fermi level. The extra electron of the CeY compounds does not change the symmetry of the ground and excited states preserving the order of magnitude of the $\Delta_{C F}$ 's when going from CeX and CeY. The slight differences in the crystal-field splittings of CeX with respect to those of CeY could be understood by the fact that the Fermi level falls in a maximum of the $5 d$-DOS in all the CeX compounds and in a valley of the $5 d$-DOS in all the CeY cases. Consequently, the $\Delta_{C F}$ 's are reduced in CeY as compared to CeX.

This work was partially funded by UBACyT-X115, PICT-0310698 and PIP 2005-2006 Num. 6016. A. M. Llois and V. L. Vildosola belong to CONICET (Argentina). 
[1] D. Adroja, et al, Phys. Rev. B 6212181 (2000)

[2] Handbook on The Physics and Chemistry of Rare Earths, Vol 15, Chapter 98, Elsevier Science Publishers B.V., 1991

[3] M. Nakayama et al, Phys. Rev. B 69155116 (2004); H. Heer, et al, J. Phys. C: Solid State Phys. 125207 (1979)

[4] N. Kioussis et al, Phys. Rev. B 4410003 (1991); B. R. Cooper et al, Phys. Rev. B 363809 (1987)

[5] R. Birgeneau et al, Phys. Rev. B 85345 (1973)

[6] O. Gunnarsson et al., Phys. Rev B 39 (1989) 1708; J. E. Han et al., Phys. Rev. Lett. 78 (1997) 939

[7] P.Blaha,K.Schwartz,G.K.H.Madsen,D.Kvasnicka and J.Luitz,WIEN2k, An Augmented Plane wave + Local Orbitals Program for Calculating Crystal Properties (Karlheinz Schwarz, Techn. Universitait Wien, Austria) 2001.ISBN 3-9501031-1-2

[8] N. Bickers et al, Phys. Rev. B 362036 (1987); N. Bickers, Reviews of Modern Phys. $59845(1987)$

[9] P. Roura-Bas, V. L. Vildosola and A. M. Llois, accepted for publication in Physica B. 


\begin{tabular}{|c|cc||c|cc|}
\hline $\mathrm{Y}$ & $\Delta_{C F}^{e x p}$ & $\Delta_{C F}^{N C A}$ & $\mathrm{X}$ & $\Delta_{C F}^{e x p}$ & $\Delta_{C F}^{N C A}$ \\
\hline $\mathrm{S}$ & 140 & 150 & $\mathrm{P}$ & 172 & 160 \\
\hline $\mathrm{Se}$ & 116 & 120 & $\mathrm{As}$ & 159 & 150 \\
\hline $\mathrm{Te}$ & 32 & 50 & $\mathrm{Sb}$ & 37 & 70 \\
\hline
\end{tabular}

Table 1: Crystal-field splittings $\Delta_{C F}$ of $\mathrm{CeX}$ and $\mathrm{CeY}$ systems. Experimental and calculated results are given in Kelvin. See Reference [3] for experimental data.

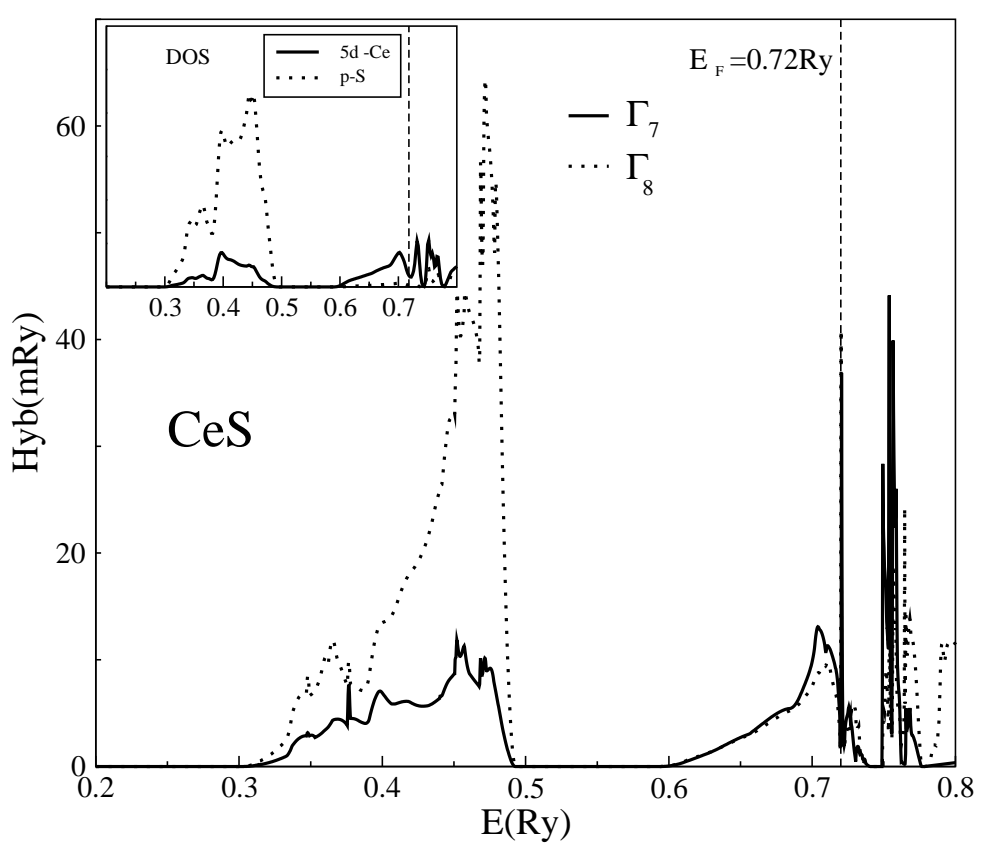

Figure 1: Calculated hybridization functions for CeS: $\Gamma_{7}$ symmetry (solid curve) and $\Gamma_{8}$ symmetry (dotted curve). Inset: $5 d-$ DOS for Ce (solid curve) and $p-$ DOS for the anion S (dotted curve). 


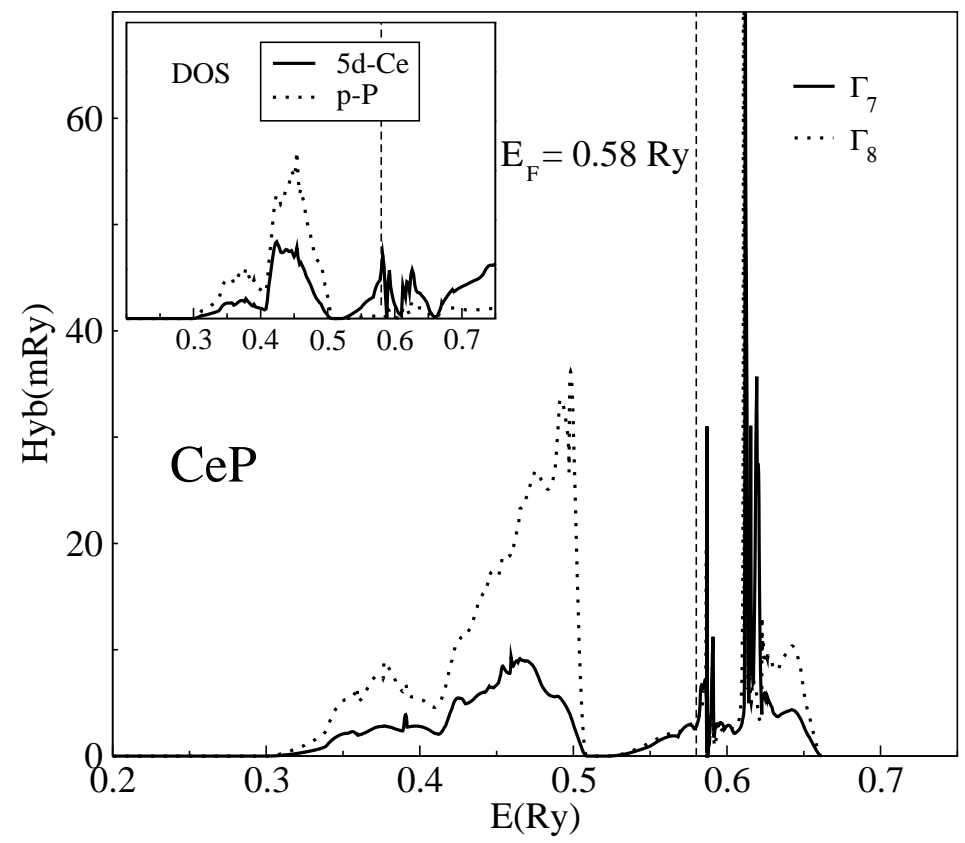

Figure 2: Calculated hybridization functions for CeP: $\Gamma_{7}$ symmetry (solid curve) and $\Gamma_{8}$ symmetry (dotted curve). Inset: $5 d-$ DOS for Ce (solid curve) and $p-$ DOS for the anion P (dotted curve). 